\title{
Erratum to: A combined treatment of hydration and dynamical effects for the modeling of host-guest binding thermodynamics: the SAMPL5 blinded challenge
}

\author{
Rajat Kumar Pal ${ }^{1,5}$ - Kamran Haider ${ }^{2}$ Divya Kaur ${ }^{6}$ William Flynn ${ }^{4,7}$. \\ Junchao $\mathrm{Xia}^{4} \cdot$ Ronald M. Levy ${ }^{4}$ - Tetiana Taran ${ }^{3}$ Lauren Wickstrom ${ }^{3}$. \\ Tom Kurtzman ${ }^{2,5,6} \cdot$ Emilio Gallicchio $^{1,5,6}$
}

Published online: 4 November 2016

(C) Springer International Publishing Switzerland 2016

\section{Erratum to: J Comput Aided Mol Des DOI 10.1007/s10822-016-9956-6}

Unfortunately, the below-mentioned errors occurred in the original publication:

1. $\Delta S_{\text {conf }}^{\circ}$ appearing before Eq. 9 has been wrongly formatted.

2. Coefficients in Tables 2, 3 and 4 for RMSE and correlation are not correctly formatted.

The original article had been corrected.

The online version of the original article can be found under doi:10.1007/s10822-016-9956-6.

Emilio Gallicchio

egallicchio@brooklyn.cuny.edu

1 Department of Chemistry, Brooklyn College, 2900 Bedford Avenue, Brooklyn, NY 11210, USA

2 Department of Chemistry, Lehman College, The City University of New York, 250 Bedford Park Blvd. West, Bronx, New York, NY 10468, USA

3 Borough of Manhattan Community College, Department of Science, The City University of New York, 199 Chambers Street, New York, NY 10007, USA

4 Center for Biophysics and Computational Biology, Institute of Computational Molecular Science and Department of Chemistry, Temple University, Philadelphia, PA, USA

5 Ph.D. Program in Biochemistry, The Graduate Center of the City University of New York, New York, NY 10016, USA

$6 \quad$ Ph.D. Program in Chemistry, The Graduate Center of the City University of New York, New York, NY 10016, USA

7 Department of Physics and Astronomy, Rutgers University, Piscataway, NJ 08854, USA
3. The values for rows G6 and G10 in Table 4 are out of their columns.

4. Figures 3 and 4 had been interchanged along with its legends.

5. Tables that are not hyperlinked in the original publication.

These errors have been corrected in the original publication. 